\title{
REVIEWS
}

\section{The Use of Low-Level Energy Laser Radiation in Basic and Clinical Research}

Department and Clinic of Internal and Occupational Diseases and Hypertension, Wroclaw Medical University, Poland

A - research concept and design; $\mathbf{B}$ - collection and/or assembly of data; C - data analysis and interpretation;

$\mathbf{D}$ - writing the article; $\mathbf{E}$ - critical revision of the article; $\mathbf{F}$ - final approval of article; $\mathbf{G}$ - other

\begin{abstract}
Laser radiation has specific attributes: monochromaticity, high coherence and polarization. These properties result in the extensive use of lasers in medicine. Laser devices can be assigned into three basic groups by means of their level of energy: high, medium and low energy. All of these types of radiation are used in medicine. However, the most commonly used, in basic science and clinical studies, is low-energy radiation. Molecular effects of low energy laser irradiation on cells are generally described as "fotobiostimulation" and "fotobiomodulation". These phenomena consequently lead to attempts to exploit this kind of radiation as a treatment method (low-level laser therapyLLLT). Areas in which LLLT is used are: regenerative medicine (for healing wounds and ulcers); aesthetic medicine (to improve appearance of scars); dentistry (to accelerate healing of implants); physiotherapy (to reduce chronic pain syndromes), orthopedics (in bone healing) and cardiology (as a prevention of restenosis after percutaneous coronary intervention). This paper discusses the medical applications of LLLT which are used in daily clinical practice as well as those used in basic science (Adv Clin Exp Med 2014, 23, 5, 835-842).
\end{abstract}

Key words: low energy laser radiation, therapy, medical application, basic science.

The laser was invented by Theodor Maiman in 1960 and since that time it is used in many different aspects of human life, including medicine. Lasers are distinguished from other light sources by their coherence, polarization and monochromaticity; therefore, they can transmit a wide range of energy.

The physical process enabling the laser to function is called stimulated emission (laser is an acronym of light amplification by stimulated emission of radiation). There are many ways to classify laser devices. Lasers can be grouped by gain medium, wavelengths, modes of operation but the most useful way, from a medical point of view, is the division by energy level. And so by the amount of energy transmitted, the sources of laser radiation are divided into 3 basic groups: low, medium and high energy.

There are many reports regarding the usefulness of full laser energy spectrum in clinical practice. High-energy radiation causes tissue destruction and therefore it is mainly applied in surgery. It is used for coagulation, cutting tissue, control of bleeding, destruction of tumors etc. Medium energy lasers are mainly used in oncology, especially as a part of photodynamic therapy. The low energy laser, among the all energy radiation groups, seems to have the widest range of applications in medicine. Despite much research, the exact mechanism action of low energy laser radiation on the human body is still unknown. Due to numerous studies on low-energy radiation, new therapeutic applications are being discovered and enrolled to clinical practice. The aim of this paper is to review the most common clinical applications of low-energy lasers.

\section{Low-Level Laser Therapy}

The use of low energy laser as a therapeutic method was first postulated by E. Master et al. [1] in 1968. Radiation used in this particular therapy refers to use the wavelength from $500 \mathrm{~nm}$ up to $1100 \mathrm{~nm}$ and the power in range from $1 \mathrm{~mW}$ to 
$500 \mathrm{~mW}$ resulting in relatively low energy density $\left(0.05 \mathrm{~J} / \mathrm{cm}^{2}-50 \mathrm{~J} / \mathrm{cm}^{2}\right)$.

Low level laser therapy (LLLT) has been clinically applied to a wide spectrum of disorders. This therapeutic method has been proven to be effective, less invasive and devoid of severe side effects for numerous diseases. Recently, a considerable amount of research is conducted to explain therapeutic mechanism of LLLT as well to disclose some new clinical applications of LLLT. Despite many studies, guidelines for this kind of treatment are still missing. For that reason, this paper summarizes the data concerning irradiation protocols and laser parameters in as much detail as it is possible.

\section{Molecular Mechanisms of LLLT Action}

Despite numerous studies, the full effect of radiation on biological reactions within the cells remains unclear. Molecular effects of low energy laser irradiation on cells are generally described as "fotobiostimulation" and "fotobiomodulation". The main manifestation of those phenomena is increased DNA and RNA synthesis [2]. This effect is achieved by modifying the number of processes occurring in the cell, the most important of which are discussed in the next part of chapter; however, a complete analysis of these modifications is beyond the scope of this paper. All discussed reactions implicate the effect of LLLT, which is most commonly used in clinical practice, namely increased cell proliferation and tissue regeneration.

\section{Changes in Retrograde Signaling}

One of the basic and most important cellular reactions is the excitation of the respiratory chain in the mitochondria, which results from photon acceptance on cytochrome $c$ oxidase, leading to increased production of reactive oxygen species (ROS) [3]. Increased level of adenosine triphosphate (ATP) synthesis and enhanced mitochondrial membrane potential $(\Delta \Psi \mathrm{m})$ are also observed as a result of laser irradiation [4]. One manifestation of these processes is an increase of cellular activity and alter cellular homeostasis parameters as well as an improvement of the intracellular signal transmission pathways, mainly those whose activity is associated with tyrosine kinase receptors (TPKR). However, recently it has been noticed that LLLT via ROS can also activate nuclear factor kappa B (NF-kB), which is a transcription factor regulating the expression of multiple gens, which can lead to a proliferation increase [5]. ROS, ATP and $\Delta \Psi \mathrm{m}$ have been described as a main part of mitochondrial retrograde signaling, which plays a crucial role in the communication between mitochondria and nucleus and has thus an important influence on cellular activities. Changes in mitochondrial retrograde signaling may lead to synthesis DNA and RNA, enzymes and proteins or changes in the membrane lipids composition. This effect of LLLT action is closely associated with the modulation of the kinases activity which affects signal transduction pathways.

\section{Modification of Kinases Activity}

Enhanced proliferation of the cells after a course of LLLT is a result of mitochondrial retrograde signaling, which exacerbates phosphorylation of TPKR, which in turn stimulates the Mitogen-activated protein kinases (MAPK)/Extracellular signal-regulated kinases (ERK) pathway [6]. TPKR affect also cell proliferation by the Phosphatidylinositide 3-kinases (PI3K)/Protein kinases B (Akt) pathway and Phospolipase C gamma (PLC-gamma)/Protein kinases C (PKC) pathway [7]. ROS produced as a cellular response to irradiation of LLLT, activates Src tyrosine kinase [8], which is responsible for increased proliferation of many kinds of cell cultures.

Low-energy laser radiation in higher doses can also induce apoptosis. The exact mechanism of this phenomenon has not been fully clarified; however, a significant relation with increased ROS production is postulated. Laser activates Glycogen synthase kinase $3 \beta$ (GSK3), which triggers apoptosis. A high-level of ROS can also lead to inactivation of the Akt/GSK3 $\beta$ signaling pathaway [9]. As a result of down regulation of this signaling pathway, greater GSK3 level is observed leading to increased apoptosis. Low energy laser radiation can induce both proliferation (by a small energy density) and apoptosis (higher energy density) by changing the activity of the same kinases, which may be observed e.g. in case of modulation of the PKC activity [10].

It appears that both apoptosis and increased proliferation after LLLT irradiation can be induced by ROS; however, the intensity of ROS production seems to be responsible for the differentiation of these 2 processes.

\section{Changes in Intracellular Cations Concentration}

Low-energy laser radiation increases the activation of the $\mathrm{Na}+/ \mathrm{K}+-\mathrm{ATPase}$ in various cells [11]. It is postulated that such activity of low-energy laser 
radiation on cells can be a putative mechanism responsible for analgesic properties of LLLT [12].

There are many reports regarding the increased intracellular level of calcium resulting from low energy laser irradiation [13]. This phenomenon might be stimulated by ROS depended mechanism [14] and via ATP depended stimuli of numerous subtypes of $\mathrm{P} 2$ purinoreceptors [15].

\section{Effectiveness of Low Energy Laser Therapy in Animal Models and Clinical Trials}

\section{Increase of Osteoblasts Proliferation}

The impact of low energy laser irradiation on molecular processes that is observed in various types of cells has become a cornerstone for designing clinical trials. Huertas et al. [16] estimated the effect of low-energy laser radiation on osteoblasts proliferation. Human osteosarcoma cell lines (MG-63) were divided into 2 groups. One underwent laser irradiation at $0.5 \mathrm{~W}, 1 \mathrm{~W}, 1.5 \mathrm{~W}$, and $2 \mathrm{~W}$ and fluencies of $1 \mathrm{~J}$ up to $5 \mathrm{~J}$ (laser wavelength was $940 \mathrm{~nm}$ ). Second group (control) was not irradiated. After $24 \mathrm{~h}$ of incubation proliferation of both groups was assessed by using a spectrophotometric measure of cell respiration (MTT assay). The authors observed a statistically significant higher proliferation in irradiated group (except of cells irradiated with high power density $2 \mathrm{~W} / \mathrm{cm}^{2}$ ). This effect was correlated with energy density and reached a peak at $3 \mathrm{~J}$ and decreased at higher fluencies. Not only is the proliferation of osteoblasts enhanced after the course of LLLT. Saygun et al. [17] demonstrated that low-energy laser irradiation can enhance growth factor production by osteoblasts. They irradiated a culture of osteoblasts with a laser $\left(685 \mathrm{~nm}, 25 \mathrm{~mW}, 14.3 \mathrm{~mW} / \mathrm{cm}^{2}\right.$, and 2 different energy densities: $2 \mathrm{~J} / \mathrm{cm}^{2}$ and $4 \mathrm{~J} / \mathrm{cm}^{2}$ ) and evaluated the release of growth factors: Basic fibroblast growth factor (bFGF), insulin-like growth factor-I (IGF-I) and receptor of insulin-like growth factor I (IGFBP3) in comparison to non-irradiated osteoblasts. All irradiated groups showed higher: proliferation, bFGF, IGF-I, and IGFBP3 expressions as compared to non-irradiation control group. Also, the group irradiated with higher energy density $\left(4 \mathrm{~J} / \mathrm{cm}^{2}\right)$ showed an increased level of bFGF and IGF-I then group with lower energy density $\left(2 \mathrm{~J} / \mathrm{cm}^{2}\right)$.

\section{Bone Healing}

Such an outcome suggests that LLLT can be used in bone healing. In literature we can find some studies which suggest that LLLT is useful in bone healing in animal models. Ribeiro et al. [18] observed better bone healing in rats treated with anti-inflammatory drugs following the course of transcutaneously LLLT (energy density $16 \mathrm{~J} / \mathrm{cm}^{2}$, power density $30 \mathrm{~W} / \mathrm{cm}^{2}$, wavelength $735 \mathrm{~nm}$ ) than no-irradiated group. In their study rats underwent surgical procedures of the bone defects in tibias. Authors relate the bone healing effect of LLLT to increased expression of cyclooxygenase-2 $(\mathrm{COX}-2)$ in fractured tissue. They postulated that LLLT can accelerate bone healing as a result of an up-regulation of COX-2 expression in bone cells. Experiment of J. Nissan et al. [19] also shows better bone healing in rats exposed to LLLT (energy density $0.72 \mathrm{~J} / \mathrm{cm}^{2}$, power density $4 \mathrm{~mW} / \mathrm{cm}^{2}$, wavelength $904 \mathrm{~nm}$ ). Rats underwent a surgical procedure of drilling an artificial cavity in their mandibules. Authors correlate better bone healing after LLLT treatment with increased calcium transport during new bone formation. Low-energy laser radiation can be useful also in bone healing after implant surgery. Khadra et al. [20] studied the effect of LLLT on bone healing around titanium implants in rabbits. They used laser with wavelength $830 \mathrm{~nm}$, energy density $23 \mathrm{~J} / \mathrm{cm}^{2}$ and power output $150 \mathrm{~mW}$. LLLT resulted in higher implant attachment in the tensile test after 8 weeks of recovery from procedure. Authors suggest that mineral analysis review increases the level of calcium and phosphorus contents on the implant surface as a result of LLLT treatment. The exact mechanism of bone healing after low-energy laser irradiation in animals is still not clear and requires further investigations in human studies.

\section{Wounds Healing}

Enhanced physiological cell proliferation after a course of low-energy laser radiation is used also in other clinical trials not only connected with bone healing. There are several papers suggesting faster healing of skin wounds or ulcers after the LLLT course [21]. Response to LLLT in wound healing models can be measured in many different ways. The most important parameter from a clinical point of view is the time needed to complete wound recovery. There are many studies that suggest that this time can be shortened by exposure on low energy laser irradiation. For example Dawood et al. [22] in their studies have shown that in rat models wound healed over 10 days earlier if they were treated with laser radiation. In their 
experiment they used a laser with a wavelength $650 \mathrm{~nm}$, power density $3.185 \mathrm{~mW} / \mathrm{cm}^{2}$ and two different energy densities: $38.2 \mathrm{~J} / \mathrm{cm}^{2}$ and $57.3 \mathrm{~J} / \mathrm{cm}^{2}$. It is worth noticing that a group irradiated with lower energy doses recovered from their wounds 2 days earlier than higher energy doses group. This suggests that an important factor in the use of LLLT as a treatment method is the proper selection of energy. Using too high-energy doses may result in the disappearance of the therapeutic effect. A similar effect of wound size reduction after low-energy laser irradiation was observed by Silveira et al. [23]. They used 2 kinds of lasers. First wavelength $904 \mathrm{~nm}$, power density $0.4 \mathrm{~W} / \mathrm{cm}^{2}$, peak power $70 \mathrm{~W}$, energy density: $1 \mathrm{~J} / \mathrm{cm}^{2}$ and $3 \mathrm{~J} / \mathrm{cm}^{2}$; second wavelength $660 \mathrm{~nm}$, power density unknown, peak power $30 \mathrm{~mW}$, energy density: $1 \mathrm{~J} / \mathrm{cm}^{2}$ and $3 \mathrm{~J} / \mathrm{cm}^{2}$. In addition, markers of oxidative stress were measured. The levels of 2-thiobarbituric acid reactive species (TBARS) were significantly decreased after irradiation of energy density $3 \mathrm{~J} / \mathrm{cm}^{2}$ on both wavelengths. Interestingly, the activity of superoxide dismutase (SOD) and catalase (CAT) was decreased in almost all irradiated groups.

Other parameters, by means of which enhanced wound healing can be measured, were investigated by Usumez et al. [24]. Authors have proved that after LLLT in many different wavelengths $(660 \mathrm{~nm}$, $810 \mathrm{~nm}, 980 \mathrm{~nm}, 1064 \mathrm{~nm}$ and energy density at level of $8 \mathrm{~J} / \mathrm{cm}^{2}$ ) in rats mucosa wound model can increase the expression of growth factor (Platelet-derived growth factor - PDGF and bFGF) in comparison to no irradiation group. Lacjaková et al. [25] observed the acceleration of fibroblast proliferation, enhanced new vessel formation and a greater amount of new collagen fibres in the wound area after laser irradiation of rat wounds model in match to non-irradiation ones.

\section{Modification of Angiogenesis}

Evaluation of angiogenesis after LLLT was the topic of Cury et al. [26] study. They examined skin flap viability in rats which underwent 8 courses of LLLT (wavelengths in this experiment were: $660 \mathrm{~nm}$ and $780 \mathrm{~nm}$, lasers fluencies: $30 \mathrm{~J} / \mathrm{cm}^{2}$ and $40 \mathrm{~J} / \mathrm{cm}^{2}$ ). It has been shown showed that LLLT (in all combination of laser parameters) can improve the healing of skin flaps by enhancing the number of new vessels formed in the tissue. This effect is probably associated with higher expression of: hypoxia inducible factor (HIF-1 $\alpha$ ), vascular endothelial growth factor (VEGF) and decrease activity of matrix metalloproteinase (MMP-2).

\section{Scar Formation}

In connection with the use of LLLT to accelerate treatment of ulcer and wound, a few clinical trials evaluating the effect of low-energy laser on scar formation were performed. Carvalho et al. [27] investigated scar formation after inguinal hernia surgery. They divided patients into 2 groups: the first underwent 4 courses (first application $24 \mathrm{~h}$ after surgery, next on days: $3,5,7$ ) of LLLT (wavelength $830 \mathrm{~nm}$, power output of $40 \mathrm{~mW}$, energy density of $13 \mathrm{~J} / \mathrm{cm}^{2}$ ). The second no-irradiation group became a control. After 6 months they evaluated scars by: vancouver scar scale (VSS), the visual analogue scale (VAS), and measurement of the scar thickness. Irradiated group showed significantly better results in the VSS, thickness measurements, malleability and pain occurrence. Similar results were observed in a study by Gaida et al. [28]. Authors evaluated the effect of 8 -week treatment by LLLT (power $400 \mathrm{~mW}$, wavelenght $670 \mathrm{~nm}$ ) in patients with burn scars. As a result of the therapy, improvement in VAS and VVS scoring was observed. There are attempts to use LLLT in treatment of old scars, but the results are not encouraging [29].

\section{Pain Reduction}

One of the most widely used applications of LLLT in clinical practice is an analgesic effect. Various biological mechanisms are involved in this effect. Bjordal et al. [30] made a systematic review of studies regarding possible mechanism of action of low-energy laser therapy in acute pain. It revealed that LLLT can modify biochemical inflammatory response by reducing the levels of prostaglandin E2 (PGE2), tumor necrosis factor (TNF), interleukin-1 (IL-1), plasminogen activator and COX-2 expression (this effect seems to be in opposition to a previously cited study Ribeiro et al., but it was confirmed in two separate studies). The same meta-analysis reveals that LLLT can also reduce: oedema and post-injury hemorrhagic formation, neutrophil cell influx, cell apoptosis and improve microcirculation. A pain reducing effect of low-energy laser irradiation has been the subject of many clinical trials. In a systematic review by Maia et al. [31] concerning patients with temporomandibular disorders (TMD) who underwent a course of low energy laser irradiation, it has been postulated that LLLT seemed to be effective in reducing pain. It should be noticed that the parameters of the laser radiation used for particular clinical trials differ significantly. Energy density was in the range from $0.9 \mathrm{~J} / \mathrm{cm}^{2}$ to $105 \mathrm{~J} / \mathrm{cm}^{2}$, power density ranged from $9.8 \mathrm{~mW}$ to $500 \mathrm{~mW}$, wavelengths contained 
in the range from $632.8 \mathrm{~nm}$ up to $904 \mathrm{~nm}$. Jang and Lee [32] in a meta-analysis of pain relief effects by laser irradiation on joint areas enclosed 22 clinical trials with over 1000 patients involved. The scope of the diseases from which patients suffered in this meta-analysis had a wide spectrum including: rheumatoid arthritis, osteoarthritis, TMD, low back pain, shoulder pain, neck pain. In studies involved in this review, parameters of laser radiation were also very divergent. Authors based on static analysis proposed most effective radiation parameters for various disorders. It should be noticed that all cited systematic reviews study confirmed the efficacy of LLLT in reducing pain caused by various diseases.

\section{LLLT in Dentistry}

Lasers have been used for a long time in dentistry and low-energy laser therapy has also been used in this domain of medicine. Except for application of LLLT mentioned above: treatment of TMD, wound healing and improving recovery after procedure of insertion of implant, LLLT is used in the treatment of dentin hypersensitivity [33]. LLLT is widely used in the prevention and treatment of oral mucositis caused by chemotherapy, radiotherapy and chemoradiotherapy used as a treatment for various types of cancer (including those outside the head and neck). Recently, there were created 2 meta-analysis consisting guidelines for dosing and preferred parameters of laser radiation. Gautam et al. [34], as a result of their meta-analysis study, made a new recommendation for the use of LLLT as a prevention of mucositis in adult patients receiving hematopoietic stem cell transplantation conditioned with high-dose chemotherapy, with or without total body irradiation. Authors suggest that those kinds of patients should receive laser radiation with a wavelength at $650 \mathrm{~nm}$, power of $40 \mathrm{~mW}$ and energy density $2 \mathrm{~J} / \mathrm{cm}^{2}$. They also suggest that LLLT in prevention of oral mucositis in patients undergoing radiotherapy head and neck cancer should have a wavelength of around $632.8 \mathrm{~nm}$. Unfortunately, in the case of other patients, no guidelines can be recommended, but it still may be assumed that LLLT is effective. Bensadoun and Nair [35] as a result of their meta-analysis recommend the use of laser irradiation in the wavelength range from $630 \mathrm{~nm}$ to $670 \mathrm{~nm}$ and from $780 \mathrm{~nm}$ up to $830 \mathrm{~nm}$, suggested power output between 10-100 $\mathrm{mW}$ and energy density $2 \mathrm{~J} / \mathrm{cm}^{2}$ up to $3 \mathrm{~J} / \mathrm{cm}^{2}$ for prophylaxis and a maximum $4 \mathrm{~J} / \mathrm{cm}^{2}$ for therapeutic effect. They assume that the treatment should be repeated each day or with a one-day break until results appear.
It is worth noting that in any of the 33 studies involved in this meta-analysis no side effects of LLLT were reported.

There are also many other clinical trials regarding the use of LLLT in dentistry. Angelov et al. [36] have used whit success LLLT in chronic advanced periodontitis. Yang and Huang [37] suggest that LLLT may have a therapeutic effect in burning mouth syndrome. The study carried out by Scoletta et al. [38] report that LLLT could be a promising modality for treating patients with bisphosphonate-induced osteonecrosis of the jaws. For details of radiation parameters, please refer to the original articles.

\section{Benefits of LLLT in Cardiology}

There are only a few reports of the use of LLLT in clinical trials in cardiology. Oron et al. [39] have investigated the effect of laser irradiation on scar formation in dog and rat models of myocardial infarction (MI). After being artificially induced with MI, animals underwent 2 courses (first course 15 min after MI, second course 3 days later) of epicardially LLLT (wavelength $803 \mathrm{~nm}$ energy density $1.08 \mathrm{~J} / \mathrm{cm}^{2}$ and a power density at level of myocardium $6 \mathrm{~mW} / \mathrm{cm}^{2}$ ). Six weeks after MI authors evaluated infarct size, it appeared that the infract size (determined by triphenyltetrazolium chloride staining technique (TTC) and histology) was significantly reduced in the group treated with LLLT in comparison to not irradiated control group. Also, the mortality rate and troponin $T$ level were decreased in the irradiated group. A histological observation of the infarct revealed a typical scar tissue in the not-irradiated group and cellularity in most of the irradiated group. Interestingly, this effect is obtained not only by direct exposure of myocardium to LLLT. Tubes et al. [40] induced artificial myocardial infarction in rats, which was followed by irradiation of the bone marrow. A significant reduction in the size of myocardial scar in the treated group was observed. Authors related this effect to the activation of autologous mesenchymal stem cells in the bone marrow. Also some other mechanism of LLLT action seems to be involved in reducing infarct size. Yaakob et al. [41] suggests that this effect in rats might be connected with an increased expression of inducible heat shock proteins (specifically heat shock protein (HSP70i)) and enhanced angiogenesis in the myocardium after laser irradiation.

Low-energy level laser irradiation is used also in clinical trials in invasive cardiology. Derkacz et al. [42] developed and patented a device for 
intravascular laser illumination. They also evaluated the usefulness of low-energy laser irradiation (wavelength $808 \mathrm{~nm}$, power density $100 \mathrm{~mW} / \mathrm{cm}^{2}$, and energy density $9 \mathrm{~J} / \mathrm{cm}^{2}$ ) of coronary arteries during percutaneous coronary intervention (PCI) in preventing restenosis. In this clinical trial 101 people were involved (52 patients in the irradiation group and 49 patients in the control group). All of the patients were with stable coronary heart disease and had undergone PCI (one group during PCI underwent a course of LLLT). After 6 months, a control angiography was performed in both groups. It was revealed that the irradiated group was characterized by a decreased rate of restenosis. Furthermore, significant differences in the minimal lumen diameter, late lumen loss, late lumen loss index were observed. In another paper, Derkacz et al. [43] have evaluated the impact of intravascular low energy laser illumination during percutaneous coronary intervention on the inflammatory process in vascular wall. Results of this study show that intravascular LLLT can significantly decrease the level of inflammatory mediators (IL $1 \beta$, IL 6) and increase the level of anti-inflammatory IL-10. Authors suggest that this may result in a decreased risk for restenosis.

The authors concluded that LLLT holds promise as a novel supportive tool in the treatment of wounds and chronic pain syndromes.

Thanks to the continuous development of laser technology and the falling costs of therapy in the future, LLLT might become a useful complementary therapy for these chronic disorders.

Nevertheless, in order to verify the usefulness of this therapeutic strategy, further clinical studies aimed both at the explanation of the therapeutic mechanisms of LLLT as well as at analyzing clinical effectiveness of such therapy in numerous disorders are needed. Appropriate laser parameters seem to be crucial in the effectiveness of this treatment method in particular disorders; therefore, future studies evaluating this issue are necessary. Although side-effects after LLLT are hardly observable, future studies of this issue are required. Due to the increased proliferative potential of the various cells after courses of LLLT, future research should be focused particularly on investigating the impact of LLLT on tumors biology.

\section{References}

[1] Mester E, Szende B, Gartner P: The effect of laser beams on the growth of hair in mice. Radiobiol Radiother 1968, 9, 621-626.

[2] AlGhamdi K, Kumar A, Moussa N: Low-level laser therapy: a useful technique for enhancing the proliferation of various cultured cells. Lasers Med Sci 2012, 27, 237-249.

[3] Karu T, Pyatibrat L, Kalendo G: Photobiological modulation of cell attachment via cytochrome c oxidase. Photochem Photobiol Sci 2004, 3, 211-216.

[4] Karu T, Pyatibrat L, Kalendo G: Irradiation with He-Ne laser increases ATP level in cells cultivated in vitro. J Photochem Photobiol 1995, 27, 219-223.

[5] Chen A, Arany P, HuangY, Tomkinson E, Sharma S, Kharkwal G, Saleem T, Mooney D, Yull F, Blackwell T, Hamblin M: Low-Level Laser Therapy Activates NF-kB via Generation of Reactive Oxygen Species in Mouse Embryonic Fibroblasts. PLoS One 2011, 6, e22453.

[6] Gao X, Xing D: Molecular mechanisms of cell proliferation induced by low power laser irradiation. J Biomed Sci $2009,16,4$

[7] Duan R, Liu T, Li Y, Guo H, Yao L: Signal transduction pathways involved in low intensity He-Ne laser-induced respiratoryburst in bovine neutrophils: a potential mechanism of low intensity laser biostimulation. Lasers Surg Med 2001, 29, 174-178.

[8] Zhang J, Xing D, Gao X: Low-power laser irradiation activates Src tyrosine kinase through reactive oxygen species-mediated signaling pathway. J Cell Physiol 2008, 217, 518-528.

[9] Huang L, Wu S, Xing D: High fluence low-power laser irradiation induces apoptosis via inactivation of Akt/ GSK3 $\beta$ signaling pathway. J Cell Physiol 2011, 226, 588-601.

[10] Gao X, Chen T, Xing D, Wang F, Pei Y, Wei X: Single cell analysis of PKC activation during proliferation and apoptosis induced by laser irradiation. J Cell Physiol 2006, 206, 441-448.

[11] Santos H, Rigos C, Tedesco A, Ciancaglini P: Biostimulation of Na,K-ATPase by low-energy laser irradiation $(685 \mathrm{~nm}, 35 \mathrm{~mW})$ : comparative effects in membrane, solubilized and DPPC:DPPE-liposome reconstituted enzyme. J Photochem Photobiol B 2007, 89, 22-28.

[12] Lopatina E, Yachnev I, Penniyaynen V, Plakhova V, Podzorova S, Shelykh T, Rogachevsky I, Butkevich I, Mikhailenko V, Kipenko A, Krylov B: Modulation of signal-transducing function of neuronal membrane $\mathrm{Na}+, \mathrm{K}+-$ -ATPase by endogenous ouabain and low-power infrared radiation leads to pain relief. Med Chem 2012, 8, 33-39.

[13] Lubart R, Friedmann H, Sinykov M, Cohen N, Breitbart H: Changes in calcium transport in mammalian sperm mitochondria and plasma membranes caused by $780 \mathrm{~nm}$ irradiation. Lasers Surg Med 1997, 21, 493-499.

[14] Lavi R, Shainberg A, Friedmann H, Shneyvays V, Rickover O, Eichler M, Kaplan D, Lubart R: Low Energy Visible Light Induces Reactive Oxygen Species Generation and Stimulates an Increase of Intracellular Calcium Concentration in Cardiac Cells. J Biol Chem 2003, 278, 40917-40922. 
[15] Kitajima S, Ozaki H, Karaki H: Role of different sub types of P2 purinoceptor on cytosolic Ca2 levels in rat aortic smooth muscle. Eur J Pharmacol 1994, 266, 263-226.

[16] Huertas R, Luna-Bertos E, Ramos-Torrecillas J, Leyva F, Ruiz C, García-Martínez O: Effect and Clinical Implications of the Low-Energy Diode Laser on Bone Cell Proliferation. Biol Res Nurs 2013, doi: 10.1177/ /1099800413482695.

[17] Saygun I, Nizam N, Ural A, Serdar M, Avcu F, Tözüm T: Low-level laser irradiation affects the release of basic fibroblast growth factor (bFGF), insulin-like growth factor-I (IGF-I), and receptor of IGF-I (IGFBP3) from osteoblasts. Photomed Laser Surg 2012, 30, 149-154.

[18] Ribeiro D, Matsumoto M: Low-level laser therapy improves bone repair in rats treated with anti-inflammatory drugs. J Oral Rehabil 2008, 35, 925-933.

[19] Nissan J, Assif D, Gross M, Yaffe A, Binderman I: Effect of low intensity laser irradiation on surgically created bony defects in rats. J Oral Rehabil 2006, 33, 619-624.

[20] Khadra M, Rønold H, Lyngstadaas S, Ellingsen J, Haanaes H: Low-level laser therapy stimulates bone-implant interaction: an experimental study in rabbits. Clin Oral Implants Res 2004, 15, 325-332.

[21] Woodruff L, Bounkeo J, Brannon W, Dawes K, Barham C, Waddell D, Enwemeka C: The efficacy of laser therapy in wound repair: a meta-analysis of the literature. Photomed Laser Surg 2004, 22, 241-247.

[22] Dawood M, Salman S: Low level diode laser accelerates wound healing. Lasers Med Sci 2013, 28, 941-945.

[23] Silveira P, Silva L, Freitas T, Latini A, Pinho R: Effects of low-power laser irradiation (LPLI) at different wavelengths and doses on oxidative stress and fibrogenesis parameters in an animal model of wound healing. Lasers Med Sci 2011, 26, 125-131.

[24] Usumez A, Cengiz B, Oztuzcu S, Demir T, Aras M, Gutknecht N: Effects of laser irradiation at different wavelengths $(660,810,980$, and $1.064 \mathrm{~nm})$ on mucositis in an animal model of wound healing. Lasers Med Sci 2013, doi: $10.1007 / \mathrm{s} 10103-013-1336-\mathrm{z}$.

[25] Lacjaková K, Bobrov N, Poláková M, Slezák M, Vidová M, Vasilenko T, Novotný M, Longauer F, Lenhardt L, Bober J, Levkut M, Sabol F, Gál P: Effects of equal daily doses delivered by different power densities of low-level laser therapy at $670 \mathrm{~nm}$ on open skin wound healing in normal and corticosteroid-treated rats: a brief report. Lasers Med Sci 2010, 25, 761-766.

[26] Cury V, Moretti A, Assis L, Bossini P, de Souza Crusca J, Neto C, Fangel R, de Souza H, Hamblin M, Parizotto N: Low level laser therapy increases angiogenesis in a model of ischemic skin flap in rats mediated by VEGF, HIF-1 $\alpha$ and MMP-2. J Photochem Photobiol B 2013, 125, 164-170.

[27] Carvalho R, Alcântara P, Kamamoto F, Cressoni M, Casarotto R: Effects of low-level laser therapy on pain and scar formation after inguinal herniation surgery: a randomized controlled single-blind study. Photomed Laser Surg 2010, 28, 417-422.

[28] Gaida K, Koller R, Isler C, Aytekin O, Al-Awami M, Meissl G, Frey M: Low Level Laser Therapy-a conservative approach to the burn scar? Burns 2004, 30, 362-367.

[29] Maluki A, Mohammad F: Treatment of atrophic facial scars of acne vulgaris by Q-Switched Nd:YAG (Neodymium: Yttrium-Aluminum-Garnet) laser 1064 nm wavelength. J Cosmet Laser Ther 2012, 14, 224-233.

[30] Bjordal J, Johnson M, Iversen V, Aimbire F, Lopes-Martins R: Low-level laser therapy in acute pain: a systematic review of possible mechanisms of action and clinical effects in randomized placebo-controlled trials. Photomed Laser Surg 2006, 24, 158-168.

[31] Maia M, Bonjardim L, Quintans Jde S, Ribeiro M, Maia L, Conti P: Effect of low-level laser therapy on pain levels in patients with temporomandibular disorders: a systematic review. J Appl Oral Sci 2012, 20, 594-602.

[32] Jang H, Lee H: Meta-analysis of pain relief effects by laser irradiation on joint areas. Photomed Laser Surg 2012, 30, 405-417.

[33] Sgolastra F, Petrucci A, Severino M, Gatto R, Monaco A: Lasers for the treatment of dentin hypersensitivity: a meta-analysis. J Dent Res 2013, 92, 492-499.

[34] Gautam A, Fernandes D, Vidyasagar M, Maiya A, Nigudgi S: Effect of low-level laser therapy on patient reported measures of oral mucositis and quality of life in head and neck cancer patients receiving chemoradiotherapy - a randomized controlled trial. Support Care Cancer 2013, 21, 1421-1428.

[35] Bensadoun R, Nair R: Low-level laser therapy in the prevention and treatment of cancer therapy-induced mucositis: 2012 state of the art based on literature review and meta-analysis. Curr Opin Oncol 2012, 24, 363-370.

[36] Angelov N, Pesevska S, Nakova M, Gjorgoski I, Ivanovski K, Angelova D, Hoffmann O, Andreana S: Periodontal treatment with a low-level diode laser: clinical findings. Gen Dent 2009, 57, 510-513.

[37] Yang H, Huang Y: Treatment of burning mouth syndrome with a low-level energy diode laser. Photomed Laser Surg 2011, 29, 123-125.

[38] Scoletta M, Arduino PG, Reggio L, Dalmasso P, Mozzati M: Effect of low-level laser irradiation on bisphosphonate-induced osteonecrosis of the jaws: preliminary results of a prospective study. Photomed Laser Surg 2010, 28, $179-184$

[39] Oron U, Yaakobi T, Oron A, Mordechovitz D, Shofti R, Hayam G, Dror U, Gepstein L, Wolf T, Haudenschild C, Ben Haim S: Low-energy laser irradiation reduces formation of scar tissue after myocardial infarction in rats and dogs. Circulation 2001, 103, 296-301.

[40] Tuby H, Maltz L, Oron U: Induction of autologous mesenchymal stem cells in the bone marrow by low-level laser therapy has profound beneficial effects on the infarcted rat heart. Lasers Surg Med 2011, 43, 401-409.

[41] Yaakobi T, Shoshany Y, Levkovitz S, Rubin O, Ben Haim S, Oron U: Long-term effect of low energy laser irradiation on infarction and reperfusion injury in the rat heart. J Appl Physiol 2001, 90, 2411-2419. 
[42] Derkacz A, Protasiewicz M, Poreba R, Szuba A, Andrzejak R: Usefulness of intravascular low-power laser illumination in preventing restenosis after percutaneous coronary intervention. Am J Cardiol 2010, 106, 1113-1117.

[43] Derkacz A, Protasiewicz M, Poręba R, Doroszko A, Andrzejak R: Effect of the intravascular low energy laser illumination during percutaneous coronary intervention on the inflammatory process in vascular wall. Lasers Med Sci 2013, 28, 763-768.

\section{Address for correspondence:}

Arkadiusz Derkacz

Department and Clinic of Internal and Occupational Diseases and Hypertension

Wroclaw Medical University

Borowska 213

50-556 Wrocław

Poland

E-mail: arkadiusz.derkacz@am.wroc.pl

Tel.: +48 717364000

Conflict of interest: None declared

Received: 17.10.2013

Revised: 13.06.2014

Accepted: 17.09.2014 